\title{
«La Belle et la Bête» au XXIe siècle et les défis d'une nouvelle vision de la masculinité: La Mécanique du coeur de Mathias Malzieu
}

\author{
Natalia GONZÁLEZ DE LA LLANA FERNÁNDEZ \\ Interkulturelle Studien- Romanistik \\ Rheinisch-Westfälische Technische Hochschule Aachen \\ natalia_llana@yahoo.com
}

\begin{abstract}
RESUME
L'article présent analyse le roman de Mathias Malzieu La mécanique du cœur comme une version moderne du conte de «La Belle et la Bête», en le comparant, en outre, avec le film de Tim Burton Edward aux mains d'argent. Ces fictions inversent toutes les deux certains des éléments de base du récit traditionnel et offrent une nouvelle vision de la masculinité.
\end{abstract}

Mots-clé: «La Belle et la Bête », La Mécanique du cœur, Mathias Malzieu, contes de fées, Edward aux mains d'argent.

[Recibido, marzo 2012; aprobado, diciembre 2012]

\begin{abstract}
The Beauty and the Beast and the challenges of a new insight on masculinity: La Mécanique du Coeur by Mathias Malzieu
\end{abstract}

\begin{abstract}
This article talks about Mathias Malzieu's novel La Mécanique du coeur, as a modern version of the fairy tale The Beauty and the Beast, comparing it as well with Tim Burton's film Edward Scissorhands. These works of fiction invert some basic elements of the traditional story and they offer a new insight on masculinity.
\end{abstract}

Keywords: The Beauty and the Beast, La Mécanique du cœur, Mathias Malzieu, fairy tales, Edward Scissorhands. 


\section{Introduction}

La mécanique du cœur de Mathias Malzieu est un roman sur l'amour, un conte de fées moderne qui a captivé de nombreux lecteurs de par le monde. Mais pour peu qu'on approfondisse sa lecture, il semble évident qu'il s'agit également d'une nouvelle version du récit de «La Belle et la Bête». Il n'est sans doute pas un héritier direct des versions populaires plus anciennes, mais il rejoint un sous-groupe plus récent de ces histoires qui ont été représentées au cinéma par des films comme La Belle et la Bête de Disney ou Edward aux mains d'argent de Tim Burton, où les personnages masculins acquièrent une plus grande place dans le déroulement de l'histoire et se dépouillent d'une grande partie de la charge négative que possédaient d'autres Bêtes précédentes.

Dans cet article, nous souhaitons analyser les éléments de La mécanique du cœur qui en permettent l'interprétation comme une recréation de «La Belle et la Bête». Pour cela, nous reviendrons tout d'abord sur l'origine de ce conte et certaines des interprétations qu'on lui a données pour nous concentrer ensuite sur le livre de Malzieu et la manière dont se manifestent les éléments particuliers de cette histoire. Enfin, nous verrons avec le film Edward aux mains d'argent comment ces deux fictions représentent des versions modernes de «La Belle et la Bête» qui inversent certains des valeurs traditionnelles du récit.

\section{2. « La Belle et la Bête »: origines et interprétations d'un conte de fées}

On ramène souvent les origines de l'histoire de «La Belle et la Bête » au récit de Cupidon et Psyché conté par l'auteur latin Apulée dans son livre L'Âne d'or, écrit au IIe siècle av. J.-C. Le dieu fait l'amour dans l'obscurité à une mortelle (Psyché) à laquelle il interdit de rechercher son identité, essayant ainsi de maintenir le secret de leur rapport et d'éviter la colère de sa mère Aphrodite, jalouse de la beauté de la femme. Mais au cours d'une visite chez ses sœurs, celles-ci la convainquent d'allumer, pendant la nuit, une bougie et de regarder son amant, car seul un monstre voudrait cacher sa véritable apparence. Lorsque, incapable de résister, elle décide de ne pas respecter l'interdiction, il s'éveille et disparaît.

Ce texte présente certains des éléments qui ont fait de la " La Belle et la Bête » un conte célèbre, comme ceux de l'amant mystérieux et menaçant, le château hanté ou les sœurs jalouses. Toutefois, le rôle de Cupidon dans ce récit se fait l'écho d'autres fiancés-animaux ou monstres qui se retrouvent dans des histoires plus anciennes, tant dans les mythes classiques de métamorphose que dans les récits chinois et hindous (Warner 1994: 274).

En tout cas, ce fut dans la France des XVIIe et XVIIIe siècles qu'eut lieu le développement littéraire de ce conte de fées selon le modèle que nous connaissons actuellement. Les femmes de l'aristocratie créèrent pour la première fois, au milieu du XVIIIe siècle, les contes de fées littéraires dans les salons, comme une espèce de jeu pour pouvoir démontrer leur intelligence et leur éducation. Selon Jack Zipes (1989 : 122), le processus pour conter une histoire à la façon d'une "bagatelle" serait le suivant: on demandait au narrateur de penser un conte fondé sur un motif particulier; on jugeait son habileté dans la mesure où il était créatif et naturel; l'audience répondait gentiment avec un compliment; un autre membre de l'audience racontait alors un autre conte, sans concurrence directe avec le premier narrateur, mais pour poursuivre le jeu et varier les possibilités d'expression linguistique. 
Ces contes qui, dans un premier moment, n'ont pas été pensés pour un public d'enfants, ont alors commencé à être écrits et publiés. Parmi eux se trouvait également « La Belle et la Bête », à qui ont été consacrées de nombreuses œuvres, comme celle de Madame de Villeneuve (1740) qui est devenue le modèle classique au XVIIIe siècle. Toutefois, ce fut celle conçue par Madame Le Prince de Beaumont en 1756 comme partie intégrante du Magasin des Enfants qui devint la version canonique de ce récit pour l'époque moderne.

Sa trame est la suivante : un riche commerçant qui a perdu sa fortune déambule dans les terres qui entourent un château hanté et cueille une fleur pour l'apporter à sa fille la plus jeune. Son acte met en colère la Bête, propriétaire du château, qui lui fait promettre que, en compensation, il va lui livrer une de ses filles. La plus jeune, prête à honorer l'engagement du père, va au palais et attend la mort, mais elle finit par discuter agréablement avec le monstre, qu'elle est, toutefois, incapable d'aimer. La jeune fille peut rendre visite à sa famille, mais reste plus longtemps que convenu et, lorsqu'à la suite d'un rêve où elle voit la Bête en train de mourir à cause de son absence, celle-ci décide de revenir pour le sauver et de se marier avec lui. Ses sentiments font que, finalement, la Bête retrouve son apparence de prince.

Madame de Beaumont a altéré l'histoire pour l'adapter à un public d'enfants, en en faisant ainsi l'un des premiers contes de fées écrits spécialement pour eux (il est impossible de donner une date exacte pour l'origine du conte de fées pour enfants, mais il est probable qu'il fît son apparition aux environs des années 20 et 30 du XVIIIe siècle). Il faut tenir compte du fait que ce récit était inclus dans un ouvrage dans lequel une gouvernante raconte une série de contes et leçons à un groupe de filles qu'elle a à sa charge. Évidemment, le rôle du conte de fées littéraire était d'instruire en charmant les plus petits des classes supérieures. Dans ce cas, on s'efforçait d'enseigner aux jeunes filles avec le récit de «La Belle et la Bête » qu’elles s'épanouissaient en refusant de s'accomplir elles-mêmes.

Les principaux messages transmis aux filles ici sont, d'après Zipes (1989: 132): a) sacrifier sa propre vie à cause des erreurs d'un père; b) apprendre à aimer un horrible homme-Bête s'il est gentil et possède de bonne manières; c) maintenir la promesse à une Bête quelles qu'en soient les conséquences. La Bête n'est en réalité pas laide parce qu'elle a de bonnes manières, c'est pourquoi les deux protagonistes sont faits l'un pour l'autre, parce qu'ils vivent conformément au code de courtoisie.

Ce conte de fées se présente, par conséquent, dans sa forme moderne, comme un instrument d'adaptation sociale, essayant plutôt de consoler les jeunes filles dominées par la peur du mariage, attendant d'être livrées par leur père à un homme qui à leurs yeux pouvaient bien ressembler à un monstre. Cette histoire les incitent à faire face au futur avec obéissance et décence, tout en maintenant l'espoir que, sous l'apparence d'un mari sauvage, puisse se trouver un bon cœur, et que l'amour féminin soit capable de transformer le côté animal de l'homme. Et, en effet, c'est un conte de transformation, mais pour les deux protagonistes, car la Belle doit apprendre à regarder au-delà des apparences, tandis que la Bête s'avère être beau au-dehors ainsi qu'à l'intérieur.

Les contes de fées traitent des limites, souvent des limites qu'impose la peur, et un de ses thèmes fondamentaux est celui d'un personnage qui sort découvrir l'inconnu et dépasse ses craintes. « La Belle et la Bête » dans ses différentes formes est un groupe de récits dont la trame se déplace d'une rencontre terrifiante avec l'autre à son acceptation ou, dans certaines versions, à son anéantissement. En tout cas, on a fait face à la menace d'un autre, on l'a exorcisé quand l'histoire s'achève. (Warner 1994: 276) 
Comme l'affirme Bruno Bettelheim (2007: 372-4), les héros et les héroïnes des contes de fées mènent une bataille comparable à celle des rites d'initiation qu'un débutant doit réaliser et qui lui permettront d'atteindre un niveau supérieur d'existence qu'il ne pouvait même pas imaginer au début de son voyage sacré. Une fois qu'il a trouvé le véritable lui-même, le héros est digne d'être aimé. Le message de ces histoires est que nous devons nous dépouiller des attitudes infantiles et en prendre d'autres plus matures si nous voulons tracer une relation intime qui promette le bonheur des deux. Ces récits indiquent que viendra un jour où nous devrons apprendre ce que nous ne savons pas encore ou, en termes psychanalytiques, que nous devrons nous débarrasser du tabou sexuel, et ce que nous avions appris comme dangereux ou horrible deviendra quelque chose de véritablement merveilleux.

C'est le cas des histoires du « cycle fiancé-animal » auquel appartient le conte de « La Belle et la Bête ». Il existe trois éléments typiques de toutes ces histoires, y compris dans ce cycle (Bettelheim 2007: 378-382). Premièrement, nous ignorons comment et pourquoi le fiancé a été transformé en un animal, malgré le fait que de nombreux contes fournissent des informations à ce sujet. Deuxièmement, il y a toujours une sorcière qui a jeté le sortilège au héros maudit et qui reste à la fin impunie. Et, finalement, c'est le père qui oblige l'héroïne à se donner à la Bête, chose que la jeune fille effectue par amour ou obéissance envers lui.

En poursuivant avec l'interprétation de Bettelheim, la méconnaissance des raisons qui ont conduit à la transformation du fiancé renvoie à un passé lointain lorsqu'on ignorait la cause de tout ce qui nous arrivait, de même que la répression sexuelle, à l'origine aussi si précoce que nous ne pouvons pas nous en souvenir. Il rappelle aussi qu'il n'y a pas longtemps encore que les parents de la classe moyenne inculquaient à leurs enfants l'idée qu'ils ne devaient pas s'intéresser au sexe jusqu'au mariage. La Bête ne peut retrouver non plus sa forme jusqu'au moment où une Belle vierge accepte de se marier avec lui.

D'un autre côté, le fait que ce soit une femme qui transforme le futur fiancé en un animal n'est pas surprenant, compte tenu du fait que ce sont nos mères ou nourrices qui, dans notre éducation la plus précoce, ont contribué à faire du sexe un tabou. La mère de la Belle est absente des contes parce que sa présence se cache derrière le déguisement de la sorcière, qui, de toute façon, n'est pas sanctionnée à la fin parce que son action (construire ce tabou autour du sexe) est, dans une certaine mesure, universelle et inévitable. La Belle, pour sa part, se lie à la Bête uniquement par amour de son père, mais, lorsque cet amour mûrit, il modifie son objet, et la jeune fille tombe amoureuse du « fiancé-animal ».

Dans la plupart des contes occidentaux, la Bête est de sexe masculin et le seul moyen de rompre le sortilège est l'amour d'une jeune fille, tandis que le type d'animal varie selon les lieux d'origine de l'histoire. Il est intéressant, dans ce sens, de remarquer comment a évolué la façon de voir les animaux tout au long des siècles. Leur menace était quelque chose de très réelle aux XVIIe et XVIIIe siècles lorsque, en temps de pénurie, les ours et les loups entraient dans les villages et les villes pour chercher de la nourriture.

$\mathrm{Au} \mathrm{XXe}$ siècle, en revanche, l'attraction pour le côté sauvage provoque une série de révisions dans le conte, de sorte que la Belle a besoin de la Bête et que la bestialité du personnage masculin est bonne, puisqu'elle enseignera quelque chose à la jeune femme. La Bête ne représente plus la menace de la sexualité ou de l'autorité masculines, mais lui montre la force de la nature qu'elle a en elle en l'invitant à l'accepter et à la 
laisser grandir. Il ne faut pas rompre le sortilège du monstre. Plutôt, il est important que la Belle apprenne à aimer la Bête qui existe à l'intérieur de l'homme pour pouvoir connaître la Bête qui existe en elle-même. Ces nouvelles versions se concentrent sur la Bête comme symbole d'une sexualité réalisée pleinement que les femmes doivent accepter pour devenir des personnes adultes normales. (Warner 1994: 307-13)

Dans les années 90 circulait déjà l'idée d'un « homme-bête racheté », libéré de l'image négative qu'il véhiculait dans les versions populaires, comme on peut le constater dans des films comme Edward aux mains d'argent (Edward Scissorhands, 1990) ou dans la version animée de La Belle et la Bête de Disney (Beauty and the Beast, 1991). La transformation qu'a subi ce conte au fil du temps est donc évidente:

In popular versions, 'Beauty and the Beast' offers a lesson in female yielding and its satisfactions. The Beast stirs desire, Beauty responds from some deep inner need which he awakens. (There are echoes here of 'Sleeping Beauty' too.) The Beast, formerly the stigmatizing envelope of the fallen male, has become a badge of the salvation he offers; Beauty used to grapple with the material and emotional difficulties of matrimony for young women; now she tends to personify female erotic pleasures in matching and mastering a man who is dark and hairy, rough and wild, and, in the psychotherapist Robert Bly's phrase, in touch with the Inner Warrior in himself.

In her encounter with the Beast, the female protagonist meets her match, in more ways than one. If she defeats him, or even kills him, if she outwits him, banishes or forsakes him, or accepts him and loves him, she arrives at some knowledge she did not possess; his existence and the challenge he offers is necessary before she can grasp it. The ancient tale of 'Cupid and Psyche' told of their love; apart from the child Pleasure whom Psyche bore, their other descendants -the tales in the Beauty and the Beast group- number among the most eloquent testaments to women's struggles, against arranged marriage, and towards a definition of the place of sexuality in love. The enchantments and disenchantments of the Beast have been a rich resource in stories women have made up, among themselves, to help, to teach, to warn. (Warner 1994: 318)

\section{La mécanique du cour: une version contemporaine de «La Belle et la Bête»}

La mécanique du cour de Mathias Malzieu commence par une citation du propre livre qui indique la clé de tous les événements de l'histoire que nous sommes sur le point de lire:

Premièrement, ne touche pas à tes aiguilles. Deuxièmement, maîtrise ta colère. Troisièmement, ne te laisse jamais, au grand jamais, tomber amoureux. Car alors pour toujours à l'horloge de ton cœur la grande aiguille des heures transpercera ta peau, tes os imploseront, et la mécanique du cœur sera brisée de nouveau. (Malzieu 2007: 9)

Ces mots, qui se présentent comme un message mystérieux, prendront pourtant tout leur sens dès les premières pages du roman. Dans une nuit glaciale du mois d'avril 1874 est né à Édimbourg Jack, notre protagoniste et narrateur du récit. Fils d'une jeune prostituée qui l'abandonne, Jack vient au monde avec l'aide de Madeleine, une sagefemme clandestine dont on dit au village qu'elle est une sorcière folle qui adore «réparer» les gens avec des prothèses mécaniques. Voyant que le nouveau-né semble avoir le cœur glacé, Madeleine décide alors de lui placer une vieille horloge coucou dans la poitrine pour le maintenir en vie. Mais pour cela, chaque matin, il lui faudra la remonter à l'aide d'une clé, sans quoi il pourrait s'endormir pour toujours. 
Bettelheim indiquait, comme nous l'avons déjà remarqué, que la transformation $\mathrm{du}$ fiancé en un "monstre » était le fait d'une sorcière qui serait le déguisement de la mère absente, et nous voyons que ce procédé est, d'une autre façon, à l'œuvre ici. La mère biologique de Jack disparaît et c'est la « sorcière » qui prend sa place en élèvant l'enfant (sorcière qui, elle, ne peut avoir d'enfants) et le transforme en une étrange créature avec un organe mécanique. Dans ce cas, toutefois, la femme recevra bien une "punition », même si elle n'est pas la conséquence directe de ses actes, puisqu'elle finira par mourir en prison.

Les limites de la prothèse que porte le garçon se dévoileront bientôt au lecteur. Le jour de son dixième anniversaire, Jack va avec Madeleine au village et y trouve la personne qui deviendra le désir et la raison de son existence: la petite Acacia, la chanteuse andalouse qui le conquerra avec sa voix et ses mouvements graciles. Le cœur du garçon ne semble malheureusement pas pouvoir supporter des émotions si intenses et l'horloge coucou devient folle devant la vision de la jeune fille. Madeleine, au contraire de la mère de laquelle nous parle Bettelheim, ne crée pas un tabou autour du sexe, mais autour de l'amour:

Tout le plaisir et toute la joie que l'amour peut faire ressentir se paient un jour ou l'autre en souffrances. [...] La mécanique de ton cœur explosera. Je t'ai moi-même greffé cette horloge, je connais parfaitement les limites de son fonctionnement. Peut-être qu'elle résisterait à l'intensité du plaisir, et encore. Mais elle n'est pas assez solide pour supporter le chagrin amoureux. (Malzieu 2007: 30)

Les mots avec lesquels nous avons vu que le livre commençait, Madeleine les écrit peu après sur une ardoise qu'elle accroche au-dessus du lit de Jack. Toutefois, il ne semble pas vouloir suivre ces conseils. Fasciné par la jeune Espagnole, qui ne quitte pas ses pensées, Jack demande à Madeleine de l'envoyer à l'école avec l'espoir de pouvoir la trouver là-bas. Malheureusement, elle ne s'y trouve pas, mais Joe si, un jeune garçon qui aime également Miss Acacia et qui tourmente notre protagoniste. Pendant quatre ans, Jack supporte ses moqueries et celles de ses autres camarades à propos de sa bizarrerie mécanique qu'il cache dans sa poitrine, jusqu'à ce qu'un jour, lors d'une bagarre, le jeune garçon blesse grièvement Joe à un œil et fuit pour ne pas être arrêté par la police. Quel meilleur moment pour aller chercher sa dulcinée en Andalousie? Au cours de son voyage, de même que pendant l'école, Jack est rejeté car il est différent, en raison toujours de son cœur mécanique. Quand, à Paris, il va chez un horloger pour qu'il ajuste sa prothèse, celui-ci le traite avec mépris:

Le ton qu'il emploie est assez dédaigneux, j'essaie pour ma part de garder mon calme. Il regarde mon horloge comme si je lui montrais quelque chose de sale.

- Je sais que vous n'êtes pas docteur! C'est une horloge classique, qu'il faut juste régler de temps en temps pour qu'elle fonctionne bien...

- Les montres sont des outils destinés à la mesure du temps, rien d'autre. Dégage d'ici avec ton harnachement diabolique. Va-t'en ou j'appelle la police !

C'était reparti comme à l'école et avec les petits couples. J'ai beau connaître cette sensation d'injustice, je ne parviendrais jamais à m'y habituer. Plus je grandis, plus c'est douloureux, au contraire. (Malzieu 2007: 59)

La condition de monstre de Jack est « confirmée » lorsque, ayant déjà atteint Grenade et ayant retrouvé Acacia, il décide de chercher un travail à l'Extraordinarium, un ancien cirque reconverti en fête foraine où l'on voit des spectacles en tout genre, le lieu 
même où la jeune fille se produit aussi. L'unique emploi libre est au train fantôme, où il manquait quelqu'un pour effrayer les passagers pendant leur trajet. La responsable n'est pas très convaincue de ses capacités quand il lui montre l'horloge, mais elle le prend quand même parce qu'elle n'a plus personne pour ce travail, non sans signaler à nouveau sa désagréable différence :

Les gens n'aiment pas les choses très différentes de ce qu'ils sont. Même s'ils en apprécient le spectacle, c'est un plaisir de voyeur. Pour eux, aller voir la femme à deux têtes revient au même qu'assister à un accident. J'ai vu beaucoup d'hommes l'applaudir, mais aucun en tomber amoureux. Ce sera pareil pour toi. (Malzieu 2007: 79)

Peu de personnes sont capables d'accepter tout naturellement l'horloge qui fait battre le cœur du garçon, et parmi elles, justement Acacia :

Pas de cris d'horreur, pas de rire. Jusqu'à présent, seuls Arthur, Anna Luna, ou Méliès n'ont pas été choqué par mon horloge-cœur. C'est un acte d'amour très important pour moi cette façon qu'elle a de me signifier. " $\mathrm{Tu}$ as un coucou entre les os ? Et alors? ». Tout simplement, si simplement... (Malzieu 2007: 87)

Au contraire d'autres « Belles », la chanteuse andalouse ne se sent à aucun moment horrifiée par la Bête, ni même a peur de lui, le méprise ou ne le tourne en ridicule. Jack, d'un autre côté, n'a rien de cette masculinité dangereuse, de cette sexualité incontrôlée que l'on voit dans les versions traditionnelles du conte. Son profil, beaucoup plus proche de celui d'Edward aux mains d'argent, comme nous le verrons par la suite, illustre les transformations d'une société moderne qui propose une nouvelle vision de la masculinité.

Et cette Bête « régénérée » est opposée ici à une autre Bête, représentée par Joe, qui apparaît soudain de nouveau dans leurs vies pour voler à la fois le travail et la fiancée du protagoniste. Joe représente à l'école la brutalité et la violence, et le fait qu'il perde un œil après s'être bagarré avec Jack, ainsi que sa corpulence physique, le font également devenir un "monstre". Par la suite, il fait tout son possible pour se retrouver avec Acacia, en s'efforçant de brouiller le couple qu'elle forme avec Jack. Il faut dire, toutefois, que l'opposition entre ces deux bêtes n'est pas aussi manichéenne que celle présentée par le film de Disney ou celle proposée dans Edward aux mains d'argent avec Jim (le fiancé de Kim), car les sentiments de Joe pour la jeune fille sont sincères et il a compris les souffrances qu'il a causées à Jack à l'école parce qu'il a dû subir lui aussi dans sa chair les mêmes réactions après la perte de son œil. (Malzieu 2007: 109-110)

Jack finira par perdre Acacia, mais la responsabilité retombe plus sur ses épaules que sur celles de son concurrent. Aveuglé par la jalousie et offensé que la jeune fille ne pense pas qu'il ait vraiment besoin de l'horloge qu'il porte sur son cœur, il arrache ses aiguilles et demande à son ami Méliès, horloger rencontré à Paris, de lui en monter une autre qui le préserve, avec laquelle il ne tombera plus jamais amoureux. Suite à l'opération, Jack tombe dans le coma et ne se réveille que trois ans plus tard. Il découvre alors qu'il aurait pu vivre sans son horloge qui n'était finalement qu'un placebo à des fins psychologiques inventé par Madeleine, probablement pour le protéger de ses propres démons, comme le font beaucoup de parents d'une manière ou d'une autre. (Malzieu 2007: 136) 
Le véritable drame de Jack est que, au contraire de ce qui se passe avec les protagonistes d'autres récits construits autour du thème de la Belle et la Bête, il ne se transforme pas, n'évolue pas, ne mûrit pas. Influencé par les craintes de Madeleine, incapable de se rendre compte que son cœur fonctionnait parfaitement et n'était pas à la merci de la prothèse qu'il portait depuis la naissance, Jack se perd dans ses rêves d'enfants en refusant inconsciemment d'accepter la réalité telle qu'elle est:

- D'après Méliès, lorsque vous vous êtes détruit le cœur sous les yeux de Miss Acacia, vous vouliez lui montrer combien vous souffriez, et du même coup combien vous l'aimiez. Un acte idiot et désespéré. Mais vous n'étiez qu'un adolescent -pire, un adolescent avec des rêves d'enfant, et qui ne peut s'empêcher de mélanger les rêves et la réalité pour survivre.

- J'étais cet adolescent-enfant il y a encore quelques minutes...

- Non, vous avez cessé de l'être en décidant d'abandonner votre ancien cœur. C'est ce que redoutait Madeleine : que vous deveniez grand. (Malzieu 2007: 138-139)

Encore obsédé par Acacia, le garçon retourne la chercher, mais ne lui dévoile pas son identité. Elle pense qu'il est mort et a épousé Joe, bien qu'elle soit encore amoureuse du souvenir de Jack. Lorsque celui lui donne son ancienne horloge pour dévoiler son identité, elle se sent alors trompée et lui dit qu'il n'existe plus pour elle. Il semble que, malgré toutes ses expériences, Jack n'ait pas appris suffisamment, il n'est pas capable de renoncer à son passé:

Mon vieux cœur, même cabossé et hors de mon corps, me fait définitivement plus rêver que le nouveau. C'est le «vrai », le mien. Et je l'ai cassé, comme un con. Qu'est-ce que je suis devenu? Un imposteur de moi-même? Une ombre transparente? (Malzieu 2007: 147)

$\mathrm{Si}$, dans les récits traditionnels de la Belle et la Bête, la femme était principalement celle qui devait évoluer jusqu'à être capable d'accepter la Bête telle qu'elle était, qui devait apprendre à mûrir en assumant la sexualité de l'âge adulte, et si la transformation de la Bête était, à chaque fois, conséquence de l'action féminine, nous notons dans $L a$ mécanique du cœur que le véritable protagoniste est la Bête, qui échoue ici dans l'amour précisément pour n'avoir pas osé quitter l'enfance, pour s'être laissé guider par la peur de la vie que lui avait inculqué la figure maternelle:

Sans l'horloge de Madeleine, tu n'aurais pas survécu au jour le plus froid du monde. Mais au bout de quelques mois ton cœur de chair et de sang se suffisait à lui-même. Elle aurait pu retirer l'horloge, comme elle le faisait avec les points de suture. Elle aurait dû, d'ailleurs. Aucune famille n'osait t'adopter à cause de ce bidouillage tic-tiquant qui sortait de ton poumon gauche. Avec le temps, elle s'est attachée à toi. Madeleine te voyait comme une petite chose fragile, à protéger à tout prix, reliée à elle par ce cordon ombilical en forme d'horloge.

Elle redoutait terriblement le jour où tu deviendrais adulte. Elle a tenté de régler la mécanique de ton cœur de façon à te garder toujours auprès d'elle. Elle nous avait promis de se faire à l'idée que tu souffrirais peut-être toi aussi de l'amour, car la vie est ainsi faite. Mais elle n'y est pas parvenue. » (Malzieu 2007: 155-156)

La répression exercée dans ce roman par la « mère »-« sorcière », en faisant un tabou, non pas du sexe mais de l'amour, devient si forte qu'elle empêche le développe- 
ment vers la maturité de Jack. Le protagoniste ne parvient pas à atteindre ici le niveau supérieur de l'existence que Bettelheim voit comme une caractéristique des contes de fées, il ne se trouve pas lui-même, n'abandonne pas les attitudes infantiles et c'est pour cela que lui est refusé ce qu'il souhaitait plus que tout: l'amour de Miss Acacia.

\section{La Bête face à une nouvelle masculinité: Edward aux mains d'argent et La méca- nique du cour}

Les similitudes qui existent entre La mécanique du cour et Edward aux mains d'argent ou même entre le style de Malzieu et de Burton furent souvent signalées dans la presse. ${ }^{1}$ Nous aimerions ici justement mettre en évidence certaines de ces similitudes ainsi que les différences, en nous concentrant notamment sur ce qui concerne le thème de la Belle et la Bête.

Les deux récits commencent par nous transporter dans le monde des contes. Le film de Burton s'ouvre avec un prologue dans lequel une vieille femme conte à sa petite-fille l'histoire de l'origine de la neige. Tout a commencé avec un homme qui avait des ciseaux pour mains parce que le vieil inventeur qui l'avait créé est décédé avant de pouvoir lui donner de vraies mains.

Le jeune Jack est né également, comme les héros, dans des conditions hors du commun, et partage avec le récit sur Edward une atmosphère de conte de fées : Il neige sur Édimbourg et un froid de canard paranormal cadenasse la ville, il pourrait s'agir du jour le plus froid du monde (Malzieu 2007: 11). Ce ne sont pas seulement les conditions climatiques qui sont extraordinaires, mais aussi le lieu de naissance de l'enfant qui le met en relation avec un personnage de légende : "Cela se passe dans une vieille maison posée en équilibre au sommet de la plus haute colline d'Édimbourg -Arthur's Seat-, un volcan serti de quartz bleu au sommet duquel reposerait la dépouille de ce bon vieux roi Arthur » (Malzieu 2007: 12)

Tous deux sont orphelins et vivent isolés du reste du monde : Edward dans son château, Jack dans sa maison sur la colline. Ils sont des "monstres" avec prothèses (c'est-àdire qu'ils sont des Bêtes non animales, mais technologiques), bien que leur origine soit différente, car alors qu'Edward a été créé par un scientifique, Jack, lui, reste humain. Leur "monstruosité", toutefois, a été très atténuée si on les compare avec d'autres Bêtes précédentes, présentant le problème d'une nouvelle définition de la masculinité. ${ }^{2}$

Le fait que ces personnages aient remplacé l'animalité par des prothèses comme source de leur différence, de leur monstruosité, n'est pas du tout secondaire. Comme l'affirme Julie Clarke, les mains d'Edward le rendent inapte pour de nombreuses tâches quotidiennes, comme ouvrir la porte, s'habiller, etc., au point qu'il se blesse même parce qu'il ne peut contrôler correctement les actions des ciseaux. Depuis la révolution industrielle, il existe la peur que les machines puissent également s'emballer et devenir incontrôlables (Clarke 2008: 97). La technologie, par conséquent, devient dans la fiction la nouvelle source de craintes.

\footnotetext{
1 Voir, par exemple, l'interview de Malzieu dans Llegir en cas d'incendi: http://www.llegirencasdincendi.es/2011/11/entrevista-con-mathias-malzieu-autor-de-metamorfosis-en-elcielo/ (consulté le 10.11.2011)

2 "Eduardo es también un texto que incide en el problema de cómo definir una nueva masculinidad para los años 90, tema que le vincula con otras películas similares estrenadas por Hollywood a principios de la década." (Martín Alegre 2000: 146)
} 
Dans le cas de Jack, cette perte de contrôle a lieu également lorsqu'il subit des émotions fortes, en particulier liées à l'amour, bien que, comme nous le constatons à la fin du roman, elle a en réalité une base psychologique, car l'horloge coucou qu'il porte dans la poitrine n'est pas vraiment nécessaire pour sa survie. Dans ce récit la perte de contrôle liée à la prothèse est donc plus métaphorique que littérale.

À la différence de Jack dont l'anomalie est le produit de son imagination, celle d'Edward est réelle. Les ciseaux qu'il a pour mains l'isolent des autres, l'empêchant ainsi d'avoir un contact physique qui lui permette de montrer son amour, comme lorsqu'il essaie de caresser le scientifique mort et lui griffe le visage, ou comme lorsque Kim lui demande de l'embrasser et qu'il répond qu'il ne peut pas. Edward est un être inachevé et solitaire avec un déficit très réel, un déficit qui est simultanément un don, car grâce à lui il peut développer une créativité illimitée qui, dans un premier temps, lui fait gagner l'admiration de tous ses voisins.

Edward commence dans une situation de solitude pour être sauvé par Peg (la mère de Kim) avec l'intention de l'intégrer et de lui offrir une famille. Ses talents pour créer de belles choses, en modelant jardins, coiffures canines ou humaines, etc., lui fournissent la brève illusion qu'il pourrait faire partie d'une société "normale", qu'il pourrait aimer une jeune fille comme Kim et peut-être même que ce sentiment pourrait être réciproque. Le rêve n'est, toutefois, que cela, simplement un rêve, car les esprits étroits des voisins finiront, lorsqu'ils seront conscients qu'ils ne peuvent pas parvenir à le mâ̂triser, par rejeter ce que tout d'abord ils avaient loué.

La seule porte de sortie d'Edward est, alors, de revenir à son château, de revenir à la solitude avec le seul réconfort de savoir qu'il a trouvé l'amour (même si c'est pour devoir l'abandonner), ayant, en outre, développé ses capacités créatrices: avant son arrivée, il n'avait jamais neigé sur la ville, mais, alors qu'il construit maintenant avec ses ciseaux de belles figures de glace, il dégage en les sculptant des flocons blancs qui retombent sur les habitants.

$\mathrm{Au}$ contraire, la situation de Jack est bien diverse. Sa différence ne correspond à aucune qualité spéciale, mais ne l'empêche pas non plus de jouir des caresses de Miss Acacia ni de l'amour en général. Elle le limite exclusivement dans la mesure où Madeleine lui a inculqué l'idée que les sentiments peuvent faire exploser son cœur mécanique et que, lorsqu'il a grandi, il n'a pas été capable de se débarrasser du mensonge hérité de sa mère adoptive. Acacia, en outre, l'accepte et l'aime depuis le début. Il ne doit pas lutter pour obtenir son affection, il ne doit pas attendre que sa Belle apprenne à le regarder autrement comme c'est le cas avec Kim et Edward. En réalité, le processus ici est le contraire, car c'est le comportement du garçon qui éloigne la jeune fille, et non son aspect ou sa différence.

Ces deux récits nous montrent de façon diverse une inversion de l'histoire de "La Belle et la Bête". Comme l'affirme Viola Rondeboom : "Interestingly, Edward Scissorhands is a "Beauty and the Beast" in reverse: Burton's story starts with Edward's socalled acceptance, but ends with his demonization". (2006: 44) Kim finit par aimer Edward, mais l'amour de la fille ne suffit pas pour contrebalancer le rejet social. Dans La mécanique du cour, d'autre part, la Belle accepte Jack depuis le début, et ce n'est pas le regard des autres, mais celui du propre protagoniste, sa distorsion de la réalité, qui l'empêche d'être avec sa bien-aimée. Les "Bêtes" ne jouissent pas dans ces œuvres d'une fin heureuse et doivent vivre en dernière instance de façon solitaire.

De même, les protagonistes de ces deux récits sont clairement les personnages masculins (Jack est même le narrateur de sa propre histoire), c'est le processus vers une 
maturité jamais atteinte qu'on nous raconte ici. Ces deux jeunes "monstres" symbolisent l'incapacité d'accepter l'altérité (le rejet des voisins envers Edward et celui de ses camarades de classe envers Jack), l'importance du regard des autres et la perception qu'on a de soi même, ainsi que l'échec dans son développement vers la maturité:

Las manos de Edward son metáforas de la incapacidad del muchacho adolescente para controlar los cambios por los que pasa su cuerpo y también de las reacciones equívocas de los demás ante esta fase transicional en la que la masculinidad aún no está bien definida. En este sentido, se puede leer la película como un fracasado rito de paso a la edad adulta, en el cual el joven no puede completar su transformación en hombre porque no hay un modelo que le pueda guiar, carencia que se debe tanto a la muerte inoportuna del Inventor como a la pasividad de los hombres del suburbio. (Martín Alegre 2002: 155)

Les personnages féminins sont présentés ici, au contraire de ce qui se passait dans d'autres versions plus anciennes de «La Belle et la Bête», comme de simples compagnons des personnages masculins, qui ne sont plus, en outre, des monstres terrifiants, mais plutôt de tendres freaks.

Pour résumer, nous pouvons affirmer que ces deux fictions invertissent et modifient certaines particularités essentielles du conte de fées en maintenant, toutefois, certains de ses motifs les plus importants. Il s'agit dans ces récits de l'histoire d'amour d'un homme "monstrueux" avec une jolie fille. Le thème du regard, de la perception de l'autre, la question de la transformation intérieur des personnages dans une recherche de maturité qui leur permette d'atteindre un niveau supérieur d'existence sont essentiels ici. Se produisent, en revanche, également, d'importantes modifications qui indiquent une évolution significative dans les relations entre hommes et femmes, et dans la construction aujourd'hui d'une nouvelle masculinité.

$\mathrm{Si}$, dans d'autres moments de l'Histoire, les jeunes femmes pouvaient craindre la rencontre avec un mari brutal et agressif, pouvaient avoir besoin d'affronter leur peur du sexe pour parvenir à développer un lien amoureux propre à l'adulte, La mécanique du cour et Edward aux mains d'argent nous présentent deux jeunes hommes charmants dépourvus de tout aspect bestial, mais peut-être aussi préoccupés de ne pas savoir comment définir leur propre masculinité. L'ancien paradigme de la masculinité représenté par Joe et Jim ne vaut évidemment pas ici : Acacia et Jim n'en veulent pas. Mais une relation avec les protagonistes (Jack et Edward) n'est finalement pas possible non plus.

Le regard des autres enlève à Edward la possibilité de s'intégrer dans la société et il doit fuir. La perception de Jack de lui-même l'empêche d'être heureux avec Miss Acacia et il reste seul. Le processus de maturation a échoué et c'est cela peut-être ce qui sépare le plus ces deux ouvrages (principalement celui de Malzieu) d'autres récits de «La Belle et la Bête».

\section{Conclusion}

Nous avons donc montré ici qu'il est possible de lire La mécanique du cæeur comme un roman s'inscrivant dans la tradition du conte de fées de «La Belle et la Bête». Les constantes recréations de cette histoire, tant dans la littérature qu'au cinéma, l'ont de manière assez prévisible éloignée de ses origines. La raison principale est à chercher dans les conceptions modernes de ce que c'est d'être un homme et une femme, conceptions qui, bien évidemment, ont subi, au long des siècles, de profondes transformations. 
Nous avons pu constater comment le personnage masculin devient le centre du récit. Ce sont ses difficultés à devenir adulte, à mûrir, qui se reflètent dans le livre de Mathias Malzieu. Si, comme l'affirme Bettelheim, le message que les contes de fées transmettent aux enfants de diverses manières est que la lutte contre les graves difficultés de la vie est inévitable, mais qu'il est toutefois possible, en faisant face, et en affrontant les privations inattendues et souvent injustes, d'arriver à maîtriser les obstacles et à s'élever, victorieux (2007: 15), La mécanique du cœur nous rappelle peut-être, alors, en tant que conte de fées moderne, avec un exemple négatif, ce qui se passe quand on refuse d'abandonner les illusions de l'enfance et de faire face à l'existence.

La peur de l'amour, de la perte de contrôle provoquée par les sentiments, est le nouveau tabou qui se construit dans ce roman. Ce héros actuel, sensible et vulnérable, qui a perdu la masculinité effrayante et agressive d'autres "Bêtes", semble avoir perdu simultanément la boussole qui lui montre le chemin à suivre, tout en restant dans une espèce de limbes adolescents qu'il n'ose pas quitter ou dont il ne sait pas comment sortir. L'ancien modèle de masculinité n'est plus valable, mais lequel doit, alors, le remplacer?

\section{Références bibliographiques}

BETTELHEIM, Bruno (2007): Psicoanálisis de los cuentos de hadas. Barcelona: Ares y Mares.

CLARKE, Julie (2008): «All Too Human : Edward Scissorhands ». Screen Education: 93-98: http://media.web.britannica.com/ebsco/pdf/419/33160419.pdf (consulté le 10.11.2011)

MALZIEU, Mathias (2007): La mécanique du cœur. Paris: Flammarion.

MARTÍN ALEGRE, Sara (2002): "La tragicomedia del monstruo en el suburbio acomodado: Eduardo Manostijeras, película de Tim Burton". Garoza: Revista de la Sociedad Española de Estudios Literarios de Cultura Popular 2: 145-164: http://webs.ono.com/garoza/G2-MartinAlegre.pdf (consulté le 10.11.2011)

RONDEBOOM, Viola (2006): American Beasts. The Cinematic Revision of Beauty and the Beast in The Elephant Man and Edward Scissorhands. Doctoraalscriptie Universiteit Utrecht: http://igitur-archive.library.uu.nl/student-theses/2007-0315200141/UUindex.html (consulté le 10.11.2011)

TENA, Patricia (2011): "Entrevista con Mathias Malzieu, autor de Metamorfosis en el cielo". Llegir en cas d'incendi:

http://www.llegirencasdincendi.es/2011/11/entrevista-con-mathias-malzieu-autorde-metamorfosis-en-el-cielo/ (consulté le 10.11.2011)

WARNER, Marina (1994): From the Beast to the Blonde: On Fairy Tales and their Tellers. New York: The Noonday Press.

ZIPES, Jack (1989): "The Origins of the Fairy Tale for Children or, How Script Was Used to Tame the Beast in Us", in Children and their Books. Gillian Avery \& Julia Briggs (eds.), pp. 119-134. Oxford: Clarendon Press. 